ORIGINAL

\title{
Estudio de la relación entre adherencia a la Escuela de la Espalda y afrontamiento del dolor en pacientes con lumbalgia crónica
}

\author{
J.L. Garcia-Arenas ${ }^{a, *}$, M.D. Rubio-Fernandez ${ }^{a}$, I. Martinez-Mir ${ }^{b}$ y E. Rubio-Gomis ${ }^{c}$ \\ a Servicio de Medicina Física y Rehabilitación, Consorcio Hospital General Universitario de Valencia, Valencia, España \\ b Dirección Gerencia-Fundación Hospital General Universitario, Consorcio Hospital General Universitario de Valencia, Valencia, \\ España \\ c Unidad de Farmacología Clínica, Departamento de Farmacología, Consorcio Hospital General Universitario de Valencia, \\ Universitat de València, Valencia, España
}

Recibido el 13 de agosto de 2018; aceptado el 16 de noviembre de 2018

\section{PALABRAS CLAVE \\ Lumbalgia crónica; Escuela de la Espalda; Adherencia; \\ Afrontamiento del dolor}

\begin{abstract}
Resumen
Introducción: La Escuela de la Espalda (EE) es un tratamiento en pacientes con lumbalgia crónica con contenidos educativos asociados a otras medidas terapéuticas como ejercicios. Es eficaz si se realiza y es bien conocido que las modificaciones en los hábitos diarios son difíciles de cumplir. Este trabajo de investigación evalúa si los pacientes que al terminar la EE tienen un predominio de estrategias activas en su afrontamiento del dolor se adhieren mejor a las enseñanzas de la EE.

Material y método: Estudio observacional prospectivo para valorar la adherencia a la EE en pacientes con dolor lumbar crónico. Se incluyeron pacientes adultos que aceptaban realizar la EE. Se excluyeron aquellos cuyo nivel cultural, enfermedad concomitante o situación personal les impidiera completar el estudio. Los pacientes fueron evaluados al inicio y tras la tercera sesión de la EE. Las variables estudiadas fueron: adherencia a los ejercicios y recomendaciones, afrontamiento al dolor, intensidad del dolor, discapacidad funcional y características sociodemográficas.

Resultados: El 67,2\% de los 116 pacientes que participaron reconocen que no cumplen diariamente con los ejercicios y enseñanzas realizadas en la EE. No se ha podido establecer relación entre los pacientes con estrategias activas de afrontamiento del dolor y la adherencia a la EE. La capacidad funcional y la intensidad del dolor no difieren entre pacientes cumplidores y no cumplidores.

Conclusiones: No se ha podido demostrar que la adherencia a los ejercicios y hábitos de la EE guarde relación con las estrategias de afrontamiento al dolor, la intensidad del dolor y la capacidad funcional de los pacientes con lumbalgia.

(c) 2018 Elsevier España, S.L.U. y SERMEF. Todos los derechos reservados.
\end{abstract}

\footnotetext{
* Autor para correspondencia.

Correo electrónico: jose-a89@hotmail.com (J.L. Garcia-Arenas)
} 


\section{KEYWORDS}

Chronic low back

pain;

Back school

programme;

Adherence;

Coping with pain
Study of the relationship between adherence to back school and coping with pain in patients with chronic low back pain

\begin{abstract}
Introduction: Back School (BS) is a treatment modality for patients with chronic low back pain with educational contents associated with other therapeutic measures such as exercise. If followed, it is effective but it is well known that it is difficult to make changes to daily habits. This study evaluated whether patients completing BS had a predominance of active strategies in coping with pain, as they adhered better to the recommendations of BS.

Material and method: Prospective observational study to evaluate BS adherence in patients with chronic low back pain. Adult patients attending BS were included in the study. Patients whose educational level, concomitant pathology or personal situation prevented them from completing the study were excluded. The patients were evaluated at the beginning and after the third session of BS. The variables studied were adherence to exercises and recommendations, coping with pain, pain intensity, functional disability and other sociodemographic characteristics.

Results: A total of $67.2 \%$ of the 116 included patients acknowledged that they did not perform the exercises and recommendations of BS every day. No relationship was established between patients with active strategies for coping with pain and adherence to BS. Functional capacity and pain intensity did not differ between adherent and non-adherent patients.

Conclusions: This study did not demonstrate that adherence to the exercises and habits taught in BS is related to coping with pain strategies, pain intensity and functional capacity in patients with chronic low back pain.

(c) 2018 Elsevier España, S.L.U. y SERMEF. All rights reserved.
\end{abstract}

\section{Introducción}

El dolor lumbar crónico es más que un síntoma con diferentes estadios de discapacidad, invalidez y cronicidad. Las evidencias sobre el diagnóstico y el tratamiento son limitadas y, además, muchos de los estudios que evalúan los tratamientos rehabilitadores conservadores no especifican las sesiones óptimas.

La total erradicación del dolor rara vez se consigue; los objetivos serán disminuirlo, mejorar la función, ayudar al paciente a retomar su actividad normal y reducir el uso innecesario de recursos sanitarios. De hecho, se aconseja que los futuros estudios incluyan el análisis de las relaciones coste-beneficio y riesgo-beneficio, así como el número óptimo de sesiones para muchos de los tratamientos conservadores ${ }^{1}$.

El American College of Physicians ha publicado una guía clínica para el diagnóstico y el tratamiento de la lumbalgia, donde reconocen la importancia de la educación del paciente y aconsejan tener en cuenta el nivel de evidencia científica que avala las diferentes técnicas terapéuticas ${ }^{2}$. Hay que enseñarle al paciente a afrontar el problema para que modifique mínimamente su vida cotidiana; es fundamental una rehabilitación activa que tenga en cuenta los factores psicosociales para realizar la correcta valoración pronóstica antes del tratamiento.

La Escuela de la Espalda (EE) es un tratamiento en grupo con contenidos educativos asociados a otras medidas terapéuticas, como ejercicios; se realiza por equipos multidisciplinares que buscan una actitud activa del paciente. No está indicada en las formas agudas. Si bien la EE realizada en el puesto de trabajo puede ser efectiva para pacientes con dolor lumbar crónico o recurrente, es poco lo que se conoce sobre su coste/efectividad y, además, hay una moderada evidencia de que reduzca el dolor y mejore la función ${ }^{1,3-5}$.

La EE realizada en nuestro entorno, con la participación activa de un psicólogo, un médico rehabilitador y fisioterapeutas, tiene una faceta de terapia psicológica cognitivo-conductual cuyo objetivo es modificar las respuestas que caracterizan las experiencias emocionales. Esta estrategia parece ser efectiva para reducir el dolor, mejorar la función y favorecer el retorno al trabajo en pacientes con lumbalgia crónica².

Las EE podrían favorecer un cambio de actitud ante el dolor de espalda, cuya intensidad no se modificaría, aunque sí habría una mejoría significativa en algunas facetas de la calidad de vida6 ${ }^{6}$.

En cualquier caso, la terapia que indiquemos al paciente solo podrá ser eficaz si se realiza, y es bien conocido que las modificaciones en los hábitos diarios son difíciles de cumplir. Así, el «cumplimiento» del paciente o la «adherencia terapéutica» son sinónimos que se pueden definir como la cooperación voluntaria del paciente para seguir las instrucciones recibidas según lo prescrito. Esto incluye momento y frecuencia ${ }^{7}$. La adherencia es un concepto dinámico que puede afectar a todos los procesos clínicos, aunque habitualmente se ha medido en relación con la terapia farmacológica.

Por otra parte, en el abordaje al paciente con dolor crónico ha cobrado importancia en las últimas décadas el afrontamiento, que ha sido definido como «los esfuerzos cognitivos y conductuales constantemente cambiantes 
que se desarrollan para manejar las demandas específicas externas $\mathrm{y} / \mathrm{o}$ internas que son evaluadas como excedentes o desbordantes de los recursos del individuo» ${ }^{8}$. Para medirlo se han utilizado numerosos cuestionarios, siendo el Cuestionario de Afrontamiento ante el Dolor Crónico un instrumento validado en la población española, de fácil aplicación, con una estructura estable y altos índices de consistencia interna, que sirve para evaluar la forma de afrontar el dolor; esta dimensión es relevante para el control y el manejo de la percepción álgica. En este cuestionario se analizan 6 estrategias de afrontamiento del dolor crónico, 2 de afrontamiento pasivo: religión y catarsis; y 4 de afrontamiento activo: distracción, autocontrol mental, autoafirmación y búsqueda de información. Los pacientes con estrategias activas, centradas en el problema, parecen presentar una mejor adaptación que aquellos con estrategias centradas en la emoción (pasivas).

En el presente trabajo de investigación queremos evaluar si los pacientes que al terminar la EE tienen un predominio de estrategias activas en su afrontamiento del dolor se adhieren mejor a las enseñanzas de la EE.

\section{Material y métodos}

\section{Diseño y ámbito del estudio y selección de la muestra}

Se realizó un estudio observacional prospectivo en pacientes adultos con dolor lumbar mecánico crónico que fueron remitidos al Servicio de Rehabilitación del Consorcio Hospital General Universitario de Valencia (CHGUV). La muestra está constituida por pacientes con dolor lumbar mecánico crónico incluidos en 16 grupos consecutivos, durante 3 años, de la EE del Servicio de Rehabilitación del CHGUV.

Los pacientes se incluyeron según los siguientes criterios de selección: mayores de 18 años con duración de los síntomas superior a 6 meses, que sepan leer y escribir y den su consentimiento informado por escrito para participar en el estudio. Los participantes fueron excluidos si tenían alguna enfermedad concomitante o una situación personal que les impidiera completar esta investigación y cuyo nivel cultural interfiriera con la comprensión o el cumplimiento del tratamiento.

\section{Variables de estudio}

La variable principal del estudio es la adherencia a las enseñanzas y las recomendaciones de la EE tras la tercera sesión de la Escuela, medida por el test de Haynes-Sackett ${ }^{9}$. Como variable independiente principal se ha registrado el afrontamiento del dolor medido con el Cuestionario de Afrontamiento del Dolor ${ }^{8}$, tanto al inicio como a la tercera sesión de la EE. Se ha considerado como estrategia principal para afrontar el dolor aquella que tiene un mayor peso en la puntuación global del cuestionario y el paciente es clasificado de acuerdo con esa estrategia prioritaria. Un paciente puede tener más de una estrategia principal si 2 o más de las 6 que se evalúan tienen la misma puntuación. Se han recogido otras variables, sociodemográficas: sexo, edad, nivel de estudios y situación laboral; y relacionadas con la enfermedad: características clínicas, grado de dolor mediante la escala visual analógica y la situación funcional medida por el test de Oswestry ${ }^{10,11}$. El nivel de estudios y la situación laboral se han clasificado de acuerdo con la Encuesta de Salud de 2003.

\section{Diseño experimental}

Los pacientes son evaluados al inicio del estudio, en la visita de inclusión, y tras la tercera sesión de la EE. La EE consiste en 4 sesiones teóricas y prácticas de $45 \mathrm{~min}$ en grupos de 10 miembros y está dirigida por un equipo multidisciplinar compuesto por un médico rehabilitador, un fisioterapeuta y un psicólogo clínico especializado en dolor; las 3 primeras sesiones se llevan a cabo en 3 semanas consecutivas y la última, de recordatorio, al mes. En el contenido teórico se exponen nociones elementales de anatomía, biomecánica corporal, posibles causas de dolor de espalda, mecanismos de protección e higiene y acción contra una crisis aguda. Se complementa con el aprendizaje de ejercicios de estiramiento, fortalecimiento, cintura pélvica $y$, finalmente, relajación. Si un paciente no asistió a alguna de las citas programadas, se hizo un esfuerzo por contactar con él o ella por teléfono para recoger las razones de ello y, si procedía, incluirlo en una nueva sesión.

\section{Análisis de los datos}

Todos los análisis estadísticos se realizaron utilizando SPSS para Windows versión 20 (IBM-SPSS, Inc., Chicago, IL, EE. UU.). Se realizó un análisis descriptivo. La relación entre las características del paciente, la adherencia a la EE y el afrontamiento del dolor tras la tercera sesión de la EE se analizó por métodos bivariados utilizando la $t$ de Student para variables cuantitativas y la prueba del chi cuadrado para variables categóricas. Se consideró una $\mathrm{p}<0,05$ para indicar la significación estadística.

El estudio fue aprobado por el Comité de Ética del CHGUV $y$ todos los pacientes firmaron el formulario de consentimiento informado.

\section{Resultados}

De un total de 187 pacientes con lumbalgia mecánica crónica referidos al Servicio de Rehabilitación y Medicina Física del CHGUV para su inclusión en la EE, cumplían los criterios de selección 154, y de ellos, 143 cumplían criterios y dieron su consentimiento para participar en el estudio. De los pacientes incluidos, 116 completaron las 3 visitas de la EE y contestaron los cuestionarios programados (fig. 1).

No hay diferencias entre las características demográficas y clínicas de los pacientes incluidos y estudiados (datos no mostrados). Las tablas 1 y 2 muestran los datos demográficos y clínicos de los 116 pacientes que finalizaron el estudio $(n=116)$. Hay que resaltar que la mayoría son mujeres en la década de los 40, con estudios primarios o superiores (en total, el $86,20 \%$ ) y laboralmente activas o amas de casa (en total, el $71,55 \%)$. En relación con su lumbalgia, la mayoría presenta un dolor de intensidad moderada que, de acuerdo con el valor del test de Oswestry, impacta de forma mayoritariamente moderada sobre la capacidad funcional. El dolor 


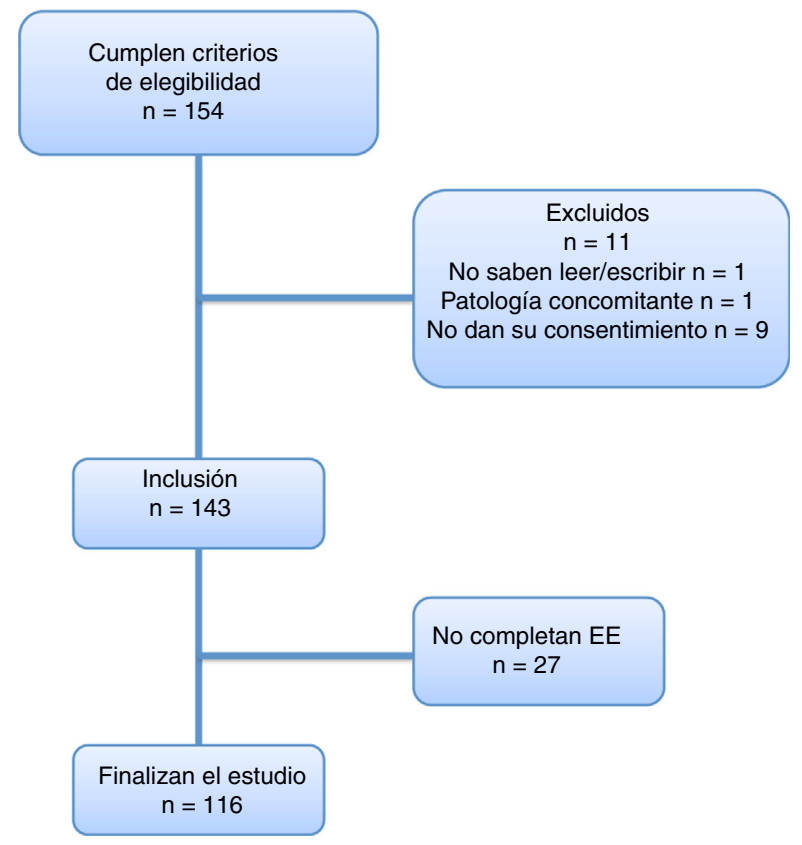

Figura 1 Diagrama de flujo.

Tabla 1 Datos demográficos de los pacientes estudiados $(\mathrm{N}=116)$

\begin{tabular}{|c|c|c|c|c|}
\hline & Media & IC $95 \%$ & $\mathrm{n}$ & $\%$ \\
\hline Edad (años) & 46,8 & $44,6-49,0$ & & \\
\hline Mujeres & & & 87 & 75 \\
\hline \multicolumn{5}{|l|}{ Nivel educativo } \\
\hline Sabe leer y/o escribir & & & 5 & 4,31 \\
\hline Primarios incompletos & & & 9 & 7,76 \\
\hline Primarios completos/EGB & & & 50 & 43,10 \\
\hline Bachiller/BUP/FP & & & 24 & 20,69 \\
\hline Universitarios & & & 26 & 22,41 \\
\hline No consta & & & 2 & 1,72 \\
\hline \multicolumn{5}{|l|}{ Situación laboral } \\
\hline Ama de casa & & & 24 & 20,69 \\
\hline Inactivo (jubilado, ILT) & & & 23 & 19,83 \\
\hline Activo & & & 59 & 50,86 \\
\hline Estudiante & & & 2 & 1,72 \\
\hline Parado (habiendo trabajado) & & & 6 & 5,17 \\
\hline Buscando primer empleo & & & 2 & 1,72 \\
\hline Oswestry ${ }^{\mathrm{a}}$ & 39,6 & $37,0-42,2$ & & \\
\hline Leve & & & 6 & 5,17 \\
\hline Moderado & & & 101 & 87,07 \\
\hline Grave & & & 9 & 7,76 \\
\hline EVA & 4,8 & $4,4-5,1$ & & \\
\hline
\end{tabular}

BUP: bachillerato unificado polivalente; EGB: educación general básica; EVA: escala visual analógica de dolor; FP: formación profesional; ILT: incapacidad laboral temporal.

a Escala de incapacidad por dolor lumbar de Oswestry.

es de inicio lento y progresivo, sin antecedente traumático, se relaciona con el movimiento y los cambios posturales y mejora con el reposo, presenta un patrón mecánico bien definido y en la mitad de los pacientes se irradia a los miembros inferiores $(53,45 \%)$.
Tras la tercera sesión de la EE, la media de dolor de los pacientes fue de 5,2 (IC 95\%4,8-5,6) y el valor medio de discapacidad funcional de acuerdo con el test de Oswestry fue de 39,6 (IC 95\% 37,0-42,2); 6 pacientes se clasificaron con discapacidad funcional leve, 101 moderada y 9 grave. 
Tabla 2 Características del dolor $(\mathrm{N}=116)$

\begin{tabular}{|c|c|c|}
\hline & $\mathrm{n}^{\mathrm{a}}$ & $\%$ \\
\hline Con antecedente traumático & 4 & 3,45 \\
\hline \multicolumn{3}{|l|}{ Forma de comienzo } \\
\hline Con episodio desencadenante & 41 & 35,34 \\
\hline Lento y progresivo & 75 & 64,66 \\
\hline \multicolumn{3}{|l|}{ Características del dolor mecánico } \\
\hline Relacionado con movimientos & 113 & 97,41 \\
\hline Relacionado con las posturas & 115 & 99,14 \\
\hline Mejora con reposo & 114 & 98,28 \\
\hline \multicolumn{3}{|l|}{ Tipo de dolor } \\
\hline Continuo & 2 & 1,72 \\
\hline Pulsátil & 1 & 0,86 \\
\hline Nocturno & 1 & 0,86 \\
\hline Irradia a miembros inferiores & 62 & 53,45 \\
\hline Hasta la rodilla & 24 & 20,69 \\
\hline Por debajo de la rodilla & 38 & 32,76 \\
\hline Con dermatoma específico & 38 & 32,76 \\
\hline Con signos neurológicos & 0 & 0,00 \\
\hline Con parestesias en MMII & 20 & 17,24 \\
\hline Con debilidad en MMII & 7 & 6,03 \\
\hline Con alteración de esfínteres & 0 & 0,00 \\
\hline Con rigidez matutina & 9 & 7,76 \\
\hline
\end{tabular}

La tabla 3 presenta las diferentes estrategias principales que los pacientes adoptan frente al dolor. Se ha considerado únicamente para cada paciente la estrategia seleccionada como principal o prioritaria. Llama la atención que más del $85 \%$ son estrategias activas tanto al inicio como al final de la EE. Nueve $(47,4 \%)$ de los pacientes que presentaban al inicio una estrategia pasiva la cambian por una activa.

De los pacientes estudiados, $80(69,0 \%)$ reconocen que no cumplen diariamente con los ejercicios y las enseñanzas realizadas en la EE. La edad media de los no adherentes a las enseñanzas de la EE es de 46,5 años (IC 95\% 43,89$49,11)$ y 22 son hombres. De acuerdo con el resultado de la tabla 4 , no se ha podido establecer relación entre los pacientes con estrategias activas de afrontamiento del dolor y la adherencia a los ejercicios y las enseñanzas de la EE.

La media de dolor en los pacientes cumplidores no difiere de la observada en los pacientes no cumplidores; tampoco se observa diferencia alguna en la media de la puntuación del test de Oswestry (tabla 5). De acuerdo con estos resultados, no se puede establecer relación alguna entre el nivel de dolor y el estado funcional con la adherencia a las enseñanzas de la EE.

\section{Discusión}

El dolor lumbar crónico tiene difícil manejo; prueba de ello son las variadas terapias que se utilizan para su tratamiento. Además, ante situaciones complejas, como es el caso de la

Tabla 3 Clasificación de los pacientes de acuerdo con la estrategia principal de afrontamiento al dolor seleccionada al inicio y tras la tercera sesión de la Escuela de la Espalda

\begin{tabular}{|c|c|c|c|c|}
\hline & \multicolumn{2}{|c|}{ Inicio del estudio } & \multicolumn{2}{|c|}{ Final del estudio } \\
\hline & $\mathrm{n}^{\mathrm{a}}$ & $\%$ & $\mathrm{n}^{\mathrm{a}}$ & $\%$ \\
\hline Total estrategias activas & 152 & 86,86 & 124 & 86,71 \\
\hline Autoafirmación & 101 & 66,45 & 77 & 62,1 \\
\hline Autocontrol & 5 & 3,29 & 12 & 9,68 \\
\hline Búsqueda de información & 34 & 22,37 & 26 & 20,97 \\
\hline Distracción & 12 & 7,89 & 9 & 7,26 \\
\hline Total estrategias pasivas & 23 & 13,14 & 19 & 13,29 \\
\hline Catarsis & 8 & 34,78 & 5 & 26,32 \\
\hline Religión & 15 & 65,22 & 14 & 73,68 \\
\hline
\end{tabular}

a Representa el número de pacientes que selecciona esa estrategia como prioritaria. El total puede ser superior a 116, dado que hay pacientes que seleccionan más de un área como prioritaria.

Tabla 4 Clasificación de los pacientes de acuerdo con la estrategia principal de afrontamiento al dolor según la adherencia a la Escuela de la Espalda

\begin{tabular}{|c|c|c|c|c|c|}
\hline \multirow[b]{3}{*}{ Estrategias } & \multicolumn{4}{|c|}{ Adherencia a la EE } & \multirow[b]{3}{*}{ Total } \\
\hline & \multicolumn{2}{|c|}{ Sí } & \multicolumn{2}{|c|}{ No } & \\
\hline & $\mathrm{n}$ & $\%$ & $\mathrm{n}$ & $\%$ & \\
\hline Activas & 29 & 80,56 & 73 & 91,25 & 102 \\
\hline Pasivas & 7 & 19,44 & 7 & 8,75 & 14 \\
\hline Total & 36 & 100 & 80 & 100 & 116 \\
\hline
\end{tabular}

EE: Escuela de la Espalda. 
Tabla 5 Pacientes adherentes o no a la Escuela de la Espalda en relación con la situación funcional e intensidad del dolor tras la Escuela de la Espalda

\begin{tabular}{|c|c|c|c|c|}
\hline & \multicolumn{2}{|c|}{ Adherencia EE } & \multicolumn{2}{|c|}{ No adherencia EE } \\
\hline & $\mathrm{n}$ & $\%$ & $\mathrm{n}$ & $\%$ \\
\hline \multicolumn{5}{|c|}{ Categorías Oswestry ${ }^{a}$} \\
\hline Leve & 3 & 8,33 & 3 & 3,57 \\
\hline Moderado & 29 & 80,56 & 72 & 90,00 \\
\hline \multirow[t]{2}{*}{ Grave } & 4 & 11,11 & 5 & 6,25 \\
\hline & $\mathrm{N}$ & Media (IC 95\%) & $\mathrm{N}$ & Media (IC 95\%) \\
\hline \multicolumn{5}{|l|}{ Puntuación } \\
\hline Oswestry $^{a}$ & 36 & $39,1(36,8-44,5)$ & 80 & $39,8(36,8-42,8)$ \\
\hline EVA & 36 & $4,6(3,8-5,5)$ & 80 & $5,5(5,1-5,8)$ \\
\hline
\end{tabular}

lumbalgia crónica inespecífica, se requiere una aproximación terapéutica multifactorial y ello es lo que pretende la $\mathrm{EE}$, que, con técnicas de terapia cognitiva, intenta modificar la conducta del paciente ante el dolor y lo sitúa en una postura activa y responsable en la prevención y el tratamiento de su dolencia. Es más, de acuerdo con Ibáñez Campos et al. ${ }^{12}$, una de las ventajas que aporta la EE es la información sobre el problema patológico y cómo modificarlo, lo que, al menos teóricamente, incidirá en la adherencia a los hábitos y las estrategias aprendidos durante su realización.

La falta de adherencia al tratamiento es considerada uno de los problemas sanitarios más extendidos en el mundo ${ }^{13}$. Está a la cabeza entre los factores que producen una mayor reducción en los resultados de salud y un incremento en los costes, principalmente asociados a recaídas y complicaciones ${ }^{14}$. En este contexto, la investigación realizada recoge los resultados sobre el grado de cumplimiento con un programa de EE y su relación con la actitud frente al dolor. Se ha considerado oportuno realizar este estudio dada la escasez de publicaciones que centran su objetivo en la adherencia a los consejos y enseñanzas de la EE, a pesar de ser bien conocido que el incumplimiento es un factor condicionante, aunque no el único, de la eficacia del tratamiento.

El perfil de paciente del presente estudio es una mujer (73,6\%), de mediana edad (46,3 años) y con estudios primarios $(41,9 \%)$, en situación laboral activa o ama de casa $(73,4 \%)$. Estas características no difieren de la población de referencia y de las descritas recientemente por BigordaSague $^{15}$ y por Bishop et al. ${ }^{16}$, aunque muestran algunas diferencias con las halladas por otros autores en pacientes que acuden a la EE o a un servicio de rehabilitación por lumbalgia crónica; en concreto, porque hay menor proporción de hombres ${ }^{17,18}$. Así, en nuestro entorno, Kovacs et al. ${ }^{19}$ y Chumillas et al. ${ }^{20}$ describen una población de iguales atributos con respecto a la edad y el nivel de estudios, pero con una mayor proporción de hombres $(47,8$ y $42,3 \%$, respectivamente); anteriormente, Ibáñez Campos et al. ${ }^{12}$ habían publicado, como características de su muestra, un valor intermedio de hombres $(32,5 \%)$ de menor edad y con predominio del grupo de bachiller (45\%). Si bien la muestra estudiada por Kovacs et al. ${ }^{19}$ es muy amplia (648 pacientes), solo una minoría presenta lumbalgia crónica $(4,3 \%)$, resultado que podría explicar las diferencias en el sexo. Es interesante resaltar en relación con el sexo que la revisión sistemática realizada por Hoy et al. ${ }^{21}$ describe que las mujeres con lumbalgia acuden al sistema sanitario más que los hombres. Es más, la intensidad del dolor y la capacidad funcional de los pacientes del presente estudio coinciden con las descritas por diferentes autores de nuestro entorno ${ }^{20,22,23}$, aunque Pinedo et al. ${ }^{6}$ muestran valores de la escala visual analógica de dolor y del test de Oswestry algo inferiores. De forma similar, las características del dolor en los pacientes del estudio coinciden al menos en parte con las referidas por Pinedo et al. ${ }^{6}$, Bigorda-Sague ${ }^{15}$ y Calvo Gutiérrez y Collantes Estévez ${ }^{24}$, y en la reciente revisión publicada en Lancet ${ }^{25}$.

De acuerdo con lo publicado por otros autores $23,26-28$, nuestros resultados al finalizar la EE muestran una reducción significativa en la escala visual analógica de dolor; sin embargo, a diferencia de otros estudios ${ }^{26,29}$, la capacidad funcional, aunque ligeramente mayor, no difiere significativamente de la observada al inicio del estudio. Por otra parte, Morata-Crespo et al. ${ }^{30}$ y Pinedo et al. ${ }^{6}$ describen la ausencia de mejoría en el test de Oswestry tras la realización de la EE, a semejanza de nuestros hallazgos. Estos autores relacionan más los resultados de la terapéutica con la percepción subjetiva de los pacientes, que con herramientas más objetivas como el test de Oswestry.

Se asume de forma general que es necesario conocer las estrategias que el paciente desarrolla frente al dolor crónico para establecer medidas que modifiquen hábitos y actitudes que conduzcan a la resolución de su enfermedad, tal como se pretende en el desarrollo de la EE. En este sentido, los resultados observados por nosotros en relación con la actitud frente al dolor coinciden con los de Soucase et al. ${ }^{31}$; la autoafirmación es la estrategia más utilizada, seguida de la búsqueda de información, entre las estrategias activas, y de las pasivas, los pacientes utilizan técnicas cognitivas o conductuales que provienen de la religión o la espiritualidad. Hay que señalar que estos autores desarrollan su investigación en pacientes con dolor crónico, aunque no son mayoritariamente pacientes con lumbalgia. Por otra parte, nuestros resultados coinciden con los descritos por BelloVillanueva et al. ${ }^{32}$, ya que los pacientes con lumbalgia crónica utilizan con mayor frecuencia estrategias adaptativas; 
sin embargo, llama la atención la alta utilización de «rezar y tener esperanza» (40\%) dentro de las estrategias pasivas, a diferencia del resultado mostrado por nosotros. Es posible que las diferencias socioculturales entre la población colombiana y la española contribuyan a explicar este resultado.

Aunque se han publicado otros artículos describiendo las estrategias de afrontamiento ante el dolor, es difícil su comparación con los resultados de esta investigación, dado que se ha establecido que las estrategias pueden variar no solo por el carácter agudo o crónico del dolor, sino también, dentro de este último, por la enfermedad que lo produce (Rodríguez Franco et al. ${ }^{33}$; Turner et al. ${ }^{34}$, Nielsen y Knardahl ${ }^{35}$ ).

Dado que la EE incide en el cambio de hábitos y actitudes, llama la atención la exigua modificación en las estrategias frente al dolor; sin embargo, hay que resaltar que más del $85 \%$ de los pacientes desarrollan estrategias activas. Los resultados obtenidos muestran la ausencia de relación entre el tipo de actitud frente al dolor y la adherencia a la EE. No es fácil discutir este hallazgo dada la escasez de publicaciones; se podría considerar que el número de pacientes con actitud pasiva frente al dolor es demasiado pequeño para poner de manifiesto relación alguna.

En nuestro estudio, el $67,2 \%$ de los pacientes reconocen que no cumplen diariamente con los ejercicios y las enseñanzas realizadas en la EE, porcentaje mayor que el publicado por otros autores, con una variación entre el 9 y el $46 \%{ }^{36,37}$. Por otra parte, Härkäpää et al. ${ }^{36}$ y Versloot et al. ${ }^{38}$ muestran evidencia de un alto grado de adhesión a la EE y cumplimiento de las normas de autocuidado. Como planteábamos en la hipótesis inicial de trabajo, aquellos pacientes con mayor adherencia deberían presentar estrategias activas frente al dolor; en ese sentido, el bajo nivel de cumplimiento con la EE mostrado en el presente estudio puede dificultar la manifestación de esta relación.

En conclusión, la ausencia de relación alguna entre estrategias activas frente al dolor y la adherencia a las enseñanzas de la EE necesita de nuevos estudios con diferente metodología que confirmen y puedan aportar otra visión que explique este resultado.

\section{Financiación}

Este trabajo ha sido realizado en parte con una ayuda para proyectos de investigación concedida por la Escola Valenciana dÉstudis de la Salut, Conselleria de Sanitat, Valencia, España (DOGV 4753 de 14/05/2004. Código PI-096/2004).

\section{Conflicto de intereses}

Los autores declaran no tener ningún conflicto de intereses.

\section{Bibliografía}

1. Airaksinen O, Brox JI, Cedraschi C, Hildebrandt J, KlaberMoffett J, Kovacs F, et al. Chapter 4. European guidelines for the management of chronic nonspecific low back pain. Eur Spine J. 2006;15:S192-300.

2. Qaseem A, Wilt TJ, McLean RM, Forciea MA. Noninvasive treatments for acute, subacute, and chronic low back pain: A clinical practice guideline from the American
College of Physicians. Ann Intern Med. 2017;166:514-30, http://dx.doi.org/10.7326/M16-2367.

3. Garcia AN, Costa Lda C, da Silva TM, Gondo FL, Cyrillo FN, Costa RA, et al. Effectiveness of back school versus McKenzie exercises in patients with chronic nonspecific low back pain: A randomized controlled trial. Phys Ther. 2013;93:729-47, http://dx.doi.org/10.2522/ptj.20120414.

4. Pieber K, Herceg M, Quittan M, Csapo R, Müller R, Wiesinger GF. Long-term effects of an outpatient rehabilitation program in patients with chronic recurrent low back pain. Eur Spine J. 2014;23:779-85.

5. Van Middelkoop M, Rubinstein SM, Kuijpers T, Verhagen AP, Ostelo R, Koes BW, et al. A systematic review on the effectiveness of physical and rehabilitation interventions for chronic non-specific low back pain. Eur Spine J. 2011;20:19-39.

6. Pinedo S, Erazo P, Betoloza JA. Escuela de la Espalda. Nuestra experiencia. Rehabilitacion (Madr). 2006;40:52-66.

7. Kripalan S, Yao X, Haynes RB. Interventions to enhance medication adherence in chronic medical conditions: A systematic review. Arch Intern Med. 2007;167:540-50.

8. Soriano J, Monsalve V. CAD: Cuestionario de Afrontamiento ante el Dolor Crónico. Rev Soc Esp Dolor. 2002;9:13-22.

9. Sackett DL, Haynes RB, Guyatt GH, Tugwell P. Cumplimiento. En: Epidemiología clínica. Ciencia básica para la medicina clínica. Madrid: Díaz de Santos; 1989. p. 250-90.

10. Flórez García M, García Pérez MA, García Pérez F, Armenteros Pedreros J, Álvarez Prado A, Martínez Lorente MD. Adaptación transcultural a la población española de la escala de incapacidad por dolor lumbar de Oswestry. Rehabilitacion (Madr). 1995;29:138-45.

11. Alcántara-Bumbiedro S, Flórez-García MT, Echávarri-Pérez C, García-Pérez F. Escala de incapacidad por dolor lumbar de Oswestry. Rehabilitacion (Madr). 2006;40:150-8.

12. Ibáñez Campos T, Carazo Dorado J, Ramos Valverde A, Arteaga Domínguez J, Prieto Lucena C, Ortega García A, et al. Escuela de espalda. Rehabilitacion (Madr). 1993;27:377-427.

13. Osterberg L, Blaschke T. Adherence to medication. N Engl J Med. 2005;353:487-97.

14. Stavropoulou C. Non-adherence to medication and doctorpatient relationship: Evidence from a European survey. Patient Educ Couns. 2011;83:7-13.

15. Bigorda-Sague A. Estudio sobre la eficacia de la escuela de espalda en la lumbalgia inespecífica. Rehabilitacion (Madr). 2012;46:222-6.

16. Bishop FL, Yardley L, Cooper C, Little P, Lewith G. Predicting adherence to acupuncture appointments for low back pain: A prospective observational study. BMC Complement Altern Med. 2017; 17, 5.

17. García-Manzanares MD, González MR, Sunyer M, Medina N, Tornero D, Limón R. Eficacia de un programa de escuela de espalda hospitalario. Rehabilitacion (Madr). 2009;43:211-7.

18. Lorenzo MA, Cáceres ML, Sánchez MD, Page A, Santos P. Eficacia de un programa de escuela de espalda. Análisis de factores asociados a la actividad laboral de los participantes. Rehabilitacion (Madr). 2011;45:233-9.

19. Kovacs FM, Fernández C, Cordero A, Muriel A, González-Luján L, Gil del Real MT, Spanish Back Pain Research Network. Non-specific low back pain in primary care in the Spanish National Health Service: A prospective study on clinical outcomes and determinants of management. BMC Health Serv Res. 2006;6:57-69.

20. Chumillas S, Peñalver L, Moreno M, Mora E. Estudio prospectivo sobre la eficacia de un programa de escuela de espalda. Rehabilitacion (Madr). 2003;37:67-73.

21. Hoy D, Brooks P, Blyth F, Buchbinder R. The epidemiology of low back pain. Best Pract Res Clin Rheumatol. 2010;24:769-81.

22. Van Tulder M, Becker A, Bekkering T, Breen A, del Real MT, Hutchinson $A$, et al. European guidelines for the management of 
acute nonspecific low back pain in primary care. Eur Spine J. 2006;15 Suppl 2:S169-91.

23. Nachemson A, Waddell G, Norlund A. Epidemiology of neck and low back pain. En: Nachemson AL, Jonsson E, editores. Neck and back pain. The scientific evidence of causes, diagnosis and treatment. Philadelphia: Lippincott Williams and Wilkins; 2000. p. $165-87$.

24. Calvo Gutiérrez J, Collantes Estévez E. Protocolo diagnóstico de la lumbalgia inflamatoria. Medicine. 2013;11:1942-4.

25. Hartvigsen J, Hancock MJ, Kongsted A, Louw Q, Ferreira ML, Genevay S, et al. What low back pain is and why we need to pay attention. Lancet. 2018;391:2356-67.

26. Skovron ML. Epidemiology of low back pain. Clin Rheumatol. 1992;3:559-73.

27. Carmona L, Ballina J, Gabriel R, Laffon A, EPISER Study Group. The burden of musculoskeletal diseases in the general population of Spain: Results from a nacional survey. Ann Rheum Dis. 2001;60:1040-5.

28. Humbría Mendiola A, Carmona L, Peña Sagredo JL, Ortiz AM. Impacto poblacional del dolor lumbar en España: resultados del estudio EPISER. Rev Esp Reumatol. 2002;29: 471-8.

29. Palomino Aguado B, Jiménez Cosmes L, Ferrero Méndez A. El dolor lumbar en el año 2009. Rehabilitacion (Madr). 2010;44:69-81.

30. Morata-Crespo AB, Tris-Ara MJ, Marín-Redondo M, RamosCarrera N, Ripoll-Muñoz E. Seguimiento de pacientes con dolor lumbar crónico tras tratamiento de escuela de espalda. Rehabilitacion (Madr). 2006;40:248-55.
31. Soucase B, Monsalve V, Soriano JF, de Andrés J. Estrategias de afrontamiento ante el dolor y calidad de vida en pacientes diagnosticados de fibromialgia. Rev Soc Esp Dolor. 2004;11:353-9.

32. Bello-Villanueva AM, Benítez-Lara M, Oviedo-Trespalacios o. Características del dolor, aspectos psicológicos, calidad de vida y estrategias de afrontamientoen pacientes con dolor de espalda crónico en una ciudad de Colombia. Rev Colomb Anestesiol. 2017;45:310-6.

33. Rodríguez Franco L, Cano García FJ, Blanco Picabia A. Evaluación de las estrategias de afrontamiento del dolor crónico. Actas Esp Psiquiatr. 2004;32:82-91.

34. Turner JA, Dworkin SF, Mancl L, Huggins KH, Truelove EL. The roles of beliefs, castrophizing, and coping in the functioning of patients with temporomandibular disorders. Pain. 2001;92:41-51.

35. Nielsen MB, Knardahl S. Coping strategies: A prospective study of patterns, stability, and relationships with psychological distress. Scand J Psychol. 2014;55:142-50.

36. Härkäpää K, Mellin G, Järvokoski A, Hurri H. A controlled study on the outcome of inpatient and autpatient treatment of low back pain. Part III. Long-term follow-up of pain, disability, and compliance. Scand J Rehabil Med. 1990;22:181-8.

37. Klaber Moffett JA, Chase SM, Portek I, Ennis JR. A controlled, prospective study to evaluate the effectiveness of a back school in the relief of chronic low back pain. Spine (Phila Pa 1976). 1986;11:120-2.

38. Versloot JM, Rozeman A, van Son AM, van Akkerveeken PF. The cost-effectiveness of a back school program in industry. A longitudinal controlled field study. Spine. 1992;17:22-7. 